1895. Mille huit cent quatre-vingt-quinze

Revue de l'association française de recherche sur

I'histoire du cinéma

$91 \mid 2020$

Ivresse patrimoniale ? - Décors d'Intolérance -

Germaine Dulac - Alice au pays des merveilles - Éric

Rondepierre

\title{
Georgina Torello, La conquista del espacio - Cine silente uruguayo (1915-1932)
}

Lilia Oliveira

\section{OpenEdition}

\section{Journals}

Édition électronique

URL : https://journals.openedition.org/1895/8070

DOI : $10.4000 / 1895.8070$

ISSN : $1960-6176$

Éditeur

Association française de recherche sur l'histoire du cinéma (AFRHC)

Édition imprimée

Date de publication : 1 juin 2020

Pagination : 182-186

ISBN : 978-2-37029-091-5

ISSN : 0769-0959

Référence électronique

Lilia Oliveira, « Georgina Torello, La conquista del espacio - Cine silente uruquayo (1975-1932)», 1895.

Mille huit cent quatre-vingt-quinze [En ligne], 91 | 2020, mis en ligne le 10 mai 2021, consulté le 01 juin 2021. URL : http://journals.openedition.org/1895/8070 ; DOI : https://doi.org/10.4000/1895.8070

Ce document a été généré automatiquement le 1 juin 2021.

(c) $\mathrm{AFRHC}$ 


\title{
Georgina Torello, La conquista del espacio - Cine silente uruguayo (1915-1932)
}

\author{
Lilia Oliveira
}

\section{RÉFÉRENCE}

Georgina Torello, La conquista del espacio - Cine silente uruguayo (1915-1932), Montevideo, Editorial Yaugurú, 2018, 275 p.

1 L'historiographie latino-américaine n'a guère eu l'habitude de donner place au cinéma muet. Cependant, depuis le Congrès de la FIAF qui eut lieu en 1982 à Oaxtepec, au Mexique, les choses commencèrent petit à petit à changer. L'éclosion éditoriale qui a accompagné la célébration du centenaire du cinéma dans le monde, au début des années 1990, augmenta l'intérêt pour le cinéma des premiers temps latino-américain. Finalement, l'avènement de la technologie digitale en tant que moyen de sauvegarder, récupérer et reproduire les films anciens fut la pièce qui manquait pour garantir une place de choix à ce cinéma. Depuis lors la prolifération d'études et de recherches a été telle que la chercheuse argentine Andrea Cuarterolo a suggéré l'expression de «Brighton latino-américain» (dans «El archivo en la época de su reproductibilidad técnica - Recursos digitales para el estudio del cine silente latinoamericando", Vivomatografías - Revista de estudio sobre precine y cine silente en Latinoamérica, $\mathrm{n}^{\circ} 3,2017$, p. 420). Elle affirme qu'en dehors de quelques publications isolées des années 1950 et 1970, les premières études centrées sur les cinémas latino-américains des premiers temps datent de la fin des années 1980 et du début des années 1990.

2 C'est dans cette période de redécouverte que prennent place les études de Georgina Torello, professeure à l'Universidad de la República Uruguay, membre du GestA - Grupo de Estudios Audiovisuales, et l'une des fondatrices de la revue électronique Vivomatografías. Son livre La conquista del espacio. Cine silente uruguayo (1915-1932), paru en 
2018 et financé par la Comisión Sectorial de Investigación Científica d'Uruguay, est centré sur le cinéma muet uruguayen. Comme dans la plupart des pays, peu de films de cette époque ont été conservés : d'après une étude réalisée en 1988 par la FIAF et la Fundación del Nuevo Cine Latinoamericano, commandé par Maria Rita Galvão, directrice de la Cinemateca Brasileira à ce moment-là, $93 \%$ des productions ibéroaméricaines du muet sont perdus. Néanmoins la chercheuse a retrouvé un ensemble de documents afférents et de scénarios concernant des œuvres considérées perdues, dont les images, les récits et même les polémiques avaient été oubliés un siècle durant. Ainsi, en effectuant l'analyse de fragments des films, d'articles de presse, de brochures, de photos des tournages, etc., elle reconstitue leurs histoires, tout en en proposant une lecture possible. Torello réalise ici un travail «archéologico-intertextuel », sur les traces de la chercheuse italienne Giuliana Bruno.

Pour ce qui est de l'organisation des matériaux, Torello a suivi la démarche de Teresa Castro et sa "raison cartographique des images». Cette notion fait référence non seulement à la présence concrète de cartes dans les productions filmiques, mais surtout à l'utilisation de "formes cartographiques", désignant les panoramiques ou vues aériennes qui permettent différents degrés de représentation et d'appropriation de l'espace (T. Castro, le Cinéma et la raison cartographique des images, Travaux de l'Institut de Géographie de Reims, $\mathrm{n}^{\circ}$ 129-130, 2007, pp. 27-37). La notion d'espace est comprise ici comme le lieu de pratiques sociales et institutionnelles, mais aussi et surtout, comme une construction visuelle et textuelle, une sorte d'artefact capable d'opérer des conquêtes autant matérielles que symboliques.

4 Partant alors des métaphores de la conquête des espaces voire de leur invasion, Torello analyse les tentatives des cinéastes locaux de capter le regard du public uruguayen par le biais de la présentation des paysages nationaux, ce qui constitue une forme de résistance à l'invasion du cinéma étranger. Le journal El Imparcial du 20 novembre 1924 annonçait, par exemple, que l'un des protagonistes du film Una niña parisiense en Montevideo était Montevideo elle-même, "notre belle ville panoramique" (p.16). Expression qui, selon l'auteure, pourrait être également appliquée, avec les ajustements nécessaires, à toute la production cinématographique uruguayenne de cette époque. Torello reconnait, pourtant, que les allusions topographiques de son livre sont également autoréférentielles, car avec cette œuvre son idée était aussi de conquérir une place dans la mémoire collective et dans l'univers académique uruguayen et mondial.

5 En dehors de l'usage des paysages pour consolider l'image d'un Uruguay moderne (à l'étranger et dans le pays), la place occupée par les femmes dans le cinéma muet uruguayen est un autre aspect mis en évidence tout au long du livre. Selon l'auteure, celles-ci n'étaient pas seulement des animatrices de séances organisées par les organisations bénévoles auxquelles elles appartenaient, mais aussi des réalisatrices, consultantes et actrices, comptant même avec un magazine dont elles étaient le publiccible - Montevideo Film (né en 1923, ce magazine n'eut que deux numéros).

6 L'année 1915 est considérée dans ce livre comme une borne marquant l'émergence d'un cinéma véritablement uruguayen. C'est, effet, en à ce moment-là qu'on commença à discuter, à un niveau institutionnel, de la fonction du cinéma en tant que diffuseur d'idées et de programmes, c'est-à-dire, à penser la cinématographie comme outil fondamental pour la domination géopolitique. C'est également cette année-là qu'il y eut, pour la première fois, une planification de la cinématographie nationale. 
7 La conquista del espacio est organisé en quatre chapitres. Le premier - «El trazado de límites. De la fecundidad progresista (a la esterilidad ejecutante) aplicada al cine » - explore la notion de proyecto (projet) en tant que geste vers le futur, comprenant la création d'utopies comme autant de stratégies de résistance. En partant des documentaires réalisés en 1915, Torello s'interroge sur les programmes partisans et étatiques visant la construction d'une cinématographie officielle.

Le premier film analysé est Transmisión del mando presidencial y gran mitin pro BattleVieira (1915), enregistrement filmé de la transmission du mandat présidentiel de José Batlle y Ordoñez à Feliciano Vieira, réalisé à l'initiative du Parti Colorado. Filmé par les opérateurs argentins Emilio et Ubaldo Peruzzi, il s'agit d'un film « capable d'enregistrer graphiquement les grandes réalisations du Parti » (El Día, 14 janvier 1915 ; cité p. 39), devenant ainsi un outil de propagande à l'intérieur et à l'extérieur de la République uruguayenne. La nouveauté était l'inclusion de la masse dans le champ, espace réservé jusque-là à l'élite du pays. Le succès de ce documentaire a ouvert le chemin à d'autres qui sont également analysés, tel La visita de Lauro Müller (1915), sur la visite du ministre des Relations extérieures du Brésil en Uruguay. Un film produit entièrement par l'État, via la récente Section photo-cinématographique du Bureau d'expositions du gouvernement, qui avait pour but de «faire connaitre le pays à l'extérieur par le biais de la production de documents fixes et en mouvement » (p. 57).

De cette même année 1915 date la première tentative de fiction uruguayenne, Artigas. Ce film, réalisé par Matilde Regalía de Roosen, d'après une initiative de Margarita U. de Herrera, présidente de la Comisión de Señoras del Comité Pro Escuelas y Patronatos (appartenant à la Liga de Damas Católicas del Uruguay), voulait développer une reconstruction épico-historique de l'indépendance de l'Uruguay. Il ne vit jamais le jour mais occupa pourtant une place importante dans la presse de Montevideo, mettant en lumière le rôle fondamental joué par les organisations bénévoles du pays dirigées par des femmes de l'élite sociale. Cette pratique se répétera plus tard dans d'autres films, tels que Pervanche (1920) et Del Pingo al volante (1929). Une preuve de la force de ce secteur de la société uruguayenne.

Ce premier chapitre s'achève sur l'analyse des projets d'Enrique Barlocco - El biógrafo, publié en 1923 - et d'Emilio Oribe - Nacionalización del séptimo arte, 1932 - centrés sur l'idée d'une monopolisation du cinéma par l'État comme seule solution à " la invasión yanqui " (l'invasion des Américains du Nord) (Oribe, Nacionalización del Séptimo Arte, Montevideo, Cuadernos de Enseñanza 2, 1932). Barlocco défendait l'idée que «le cinématographe pourrait servir à l'étranger comme un précieux moyen de propagande, le plus expressif, le plus simple, le plus sympathique et même le meilleur marché " (E. Barlocco, El Biógrafo. Academia de los Inventos, Montevideo, Imp. La Uruguaya, 1923, cité par Torello, p. 73). Finalement, ces projets ne furent jamais mis à exécution. Ces échecs dans la construction d'une cinématographie d'État laissèrent le domaine aux mains de l'initiative privée.

11 Le deuxième chapitre - «El discreto encanto de la (in)movilidad: la linterna mágica en el programa cinematográfico »- tourne autour de la notion d'intermédialité et de ses implications pour le cinéma national. Il relève une pratique assez commune à Montevideo au début $\mathrm{du} \mathrm{xx}^{\mathrm{e}}$ siècle, la projection d'images fixes nationales (photographies) mêlées aux images en mouvement étrangères (films). Ces projections, héritières de la lanterne magique, étaient mises en place lors de matinées organisées par des organisations bénévoles qui demeurèrent actives jusqu'à la moitié des années 1920. 
L'auteure affirme n'avoir pas trouvé à ce jour de semblable pratique dans d'autres pays du continent américain. Ces projections fonctionnaient comme un outil de propagande pour ces organisations, tout en étant un geste d'inclusion du national dans un secteur dominé par l'étranger. Ce national donnait évidemment une représentation de l'élite sociale du pays, de ses hobbies, ses activités, ses quartiers, etc. En même temps, Torello attire l'attention sur le fait que l'incorporation de cette pratique autochtone permet de réfléchir à la coexistence entre fixe et animé, entre la photographie nationale et les productions étrangères et, d'une certaine façon, à la résistance à l'invasion étrangère par la société uruguayenne. En raison de la perte presque totale des matériels projetés, le fil rouge, pour cette partie de la recherche, tient aux informations trouvées dans la presse. L'auteure relève ici les difficultés que l'instabilité du mot «vistas» (vues) parfois «film» ou «retrato» (portrait) - impose à sa méthodologie. "Comment distingue-t-on dans ce qui est rapporté dans la presse entre ce qui correspond à la production cinématographique et la production photographique ?» (p. 84), s'interroget-elle. Cependant, ce qui, en principe, semblait être un labyrinthe ou au mieux un obstacle, est devenu la marque la plus évidente de la vocation intermédiale entre projection et réception développée en Uruguay à l'époque du muet.

Le troisième chapitre - "Exhibiciones y camuflajes. Las ficciones topofilicas de la elite »nous plonge dans l'univers de la fiction, centré sur la construction filmique d'un Uruguay moderne et cosmopolite, exploitant l'idée de substitut territorial comme forme particulière - voire paradoxale - de conquête de l'espace cinématographique national. Ici Torello nous fait remarquer également le déplacement qui s'opère du plaisir littéraire des Uruguayens vers le plaisir voyeuriste offert par le cinéma. L'homme à la caméra devient une allégorie involontaire du regard distancié et fasciné, relevée par les érudits de la cinématographie nationale dès les débuts.

Des trois films analysés - Pervanche (1920), réalisé par l'Argentin León Ibañez Saavedra, Una niña parisiense en Montevideo (1924), du Français Georges M. de Neuville, et Del Pingo al volante (1929), de l'Uruguayen Robert Kouri-, seul le dernier a été conservé, sous forme d'une copie teintée avec intertitres originaux. Il s'agit d'un film montrant un groupe social de l'élite qui semble n'avoir plus besoin d'être ailleurs, comme c'était le cas des autres deux productions traitées. L'enjeu y est plutôt d'atténuer la dichotomie entre la campagne et la ville, autre thème occupant l'imaginaire uruguayen depuis des décennies.

Dans le cas de Pervanche, la publication de l'intrigue et de quelques photos de tournage dans la presse sont les rares documents retrouvés. Ce film, comme Artigas, fut planifié et promu par l'organisation bénévole, Entre Nous, qui s'occupa même de choisir le casting, composé par des membres de l'élite de Montevideo, relativement habitués à poser devant la caméra dans les « vues » uruguayennes. Les manoirs des familles aisées sont utilisés comme décor. Le titre, emprunté au roman français homonyme de l'écrivaine Gyp (Pervenche, 1904), et l'histoire, adaptée de Mademoiselle de la Seiglière de Jules Sandeau (1848), offrent, selon Torello, une bonne métaphore du processus d'appropriation de la culture étrangère par l'élite vernaculaire alors sous l'influence de la culture française. L'histoire se passe en France mais, filmée en Uruguay, elle révèle un espace polyvalent à lire à la fois comme un moyen concret de visualisation du pittoresque uruguayen et de représentation de l'espace français.

Une niña parisiense en Montevideo, dont le réalisateur était français, corrobore cette importance de l'influence française. Poussé par la victoire de l'équipe de football 
uruguayenne aux Jeux olympiques de Paris de 1924 et par le désir de montrer aux étrangers que l'Uruguay n'était pas une terre de sauvages, le film est structuré sur deux niveaux: celui d'un Uruguay exotique et dangereux, présenté dans les fantasmes oniriques de la protagoniste, et celui de l'Uruguay contemporain, dépeint comme un pays moderne et développé. Cette double représentation du pays assimile la dichotomie civilisation-barbarie, présente dans les discours hégémoniques civilisateurs et positivistes de l'époque, tout en établissant des compromis, voire une alliance avec l'État dans son projet de construction de la Nation.

Finalement, dans le dernier chapitre - «Del margen al centro: conventillos y rancheríos como horizonte »-, Torello se plonge dans des films qui dépeignent l'univers marginal de la société uruguayenne en ce début de siècle. On n'est plus dans la capitale riche et européanisée comme dans les autres films analysés auparavant. Il s'agit cette fois d'un cinéma qui n'hésite pas à faire voir les exclus du pays, en montrant le conventillo (maison collective habitée par les pauvres), la ferme et la vie à la campagne. L'auteure examine alors l'émergence d'une poétique de la marge dans le cinéma national qui n'abandonne pourtant pas un discours visuel (au cinéma) ou textuel (dans la presse) promouvant toujours un Uruguay peint aux couleurs des élites.

Les films analysés dans ce chapitre - Puños y nobleza (1921) de Rebaglietti, Almas de la costa (1924) de Juan A. Borges, El departamento de Treinta y Tres (1932) et El pequeño héroe del Arroyo de Oro (1932) de Carlos Alonso, ce dernier seul encore conservé - inaugurent une cinématographie qui détourne le discours filmique de sa position eurocentrée et cosmopolite, tout en inaugurant une poétique de la marge, plus uruguayenne que jamais.

18 Bien que l'on trouve une filmographie riche et détaillée du cinéma uruguayen entre 1915 et 1932 en fin de volume, La Conquista del Espacio n'est en rien une œuvre de compilation des films réalisés en Uruguay dans les premières décennies $\mathrm{du} \mathrm{xx}^{\mathrm{e}}$ siècle. Ce livre dresse un véritable état des lieux du cinéma en Uruguay au début du siècle, ainsi que de sa société, allant des prises de décisions politiques aux coutumes de son élite sociale, sans négliger les classes dominées, celles-ci, quoique moins représentées, étant tout de même présentes dans les films de cette époque. Avec cet ouvrage, Georgina Torello rend visible l'histoire du cinéma de son pays à l'époque du muet, présentant des nombreuses découvertes qui confirment l'étonnante fécondité d'un cinéma encore mal connu. 